\title{
Exploiting Parallelism for Energy Efficient Source Code High Performance Computing
}

\author{
Naeem Zafar Azeemi ${ }^{\dagger}$ \\ Christian Doppler Laboratory for Design Methodology of Signal Processing Algorithms, \\ Institute of Communications and Radio Frequency Engineering, University of Technology Vienna, \\ Gusshausstrasse 25/389, A-1040 Vienna Austria \\ Email: nzafar@nt.tuwien.ac.at
}

\begin{abstract}
Architectures are increasingly becoming difficult to fully utilize. The growing trend towards the multiple peripherals on single chip complex embedded system having multiple peripherals has fueled the energy aspect of compute and data intensive applications. Our experiments show that although high performance applications tends to be more cycle efficient, but there energy efficiency is reduced by many factors, such as optimal architecture utilization, poor compilation optimization, to name a few. Our methodology exploits parallelism, inherent in multimedia DSP applications, as well as in multimedia DSP processors. Our proposed techniques include profile based compilation-approach which makes the source-to-source transformation more energy efficient. The profile monitor identifies the application expression slacks with respect to the underlying hardware architecture in order to selectively apply different transformation schemes depending on the observed static and runtime profile and to filter out unnecessary optimization iteration. We also propose a stochastic filtering technique to further reduce the optimization search space and hence offline compilation overhead due to huge compiler optimization options. Our experiments show that the proposed techniques increase the parallelism by close to $51 \%$ for Viterbi decoder, $79 \%$ for MPEG-2, 32\% for $H-263$, and $84 \%$ for MPEG-4 without loosing performance benefits.
\end{abstract}

\section{Keywords:}

Embedded Applications, Low Energy, HPC (High Performance Computing), Genetic Algorithm, Source-toSource Transformation (StS).

\footnotetext{
${ }^{\dagger}$ This work has been funded by Chritian Doppler Laboratory for Design Methodology of Signal Processing
}

\section{Introduction}

HPC are increasingly becoming lucrative choice for parallel architectures both at architecture level as well as application level. In the same vein, energy consumption issue is steering the 90's design paradigm shift. The decrease in off-the-shelf hardware and increase in independent software vendors (ISV) has encouraged the system designer consideration. The demand for handheld multimedia applications has exploded in the recent years. In the same vein persistent increase in computer performance has been accompanied by a commensurate increase in energy dissipation. The energy efficiency of these systems today depends heavily on their software design [1]. As a result the efficient source code and energy consumption optimization has become the primary requirement of embedded system-level design methodologies. In energy sensitive scenario it is vital to research new energy optimization techniques, which should focus on optimizing energy saving while keeping performance constraints such as execution cycles and architectural usage $[2,3,4,5]$.

The tradeoff to consider energy issue at both application and architecture level includes the offline time of compilation and optimization [5, 6, 9]. Knowing the fact, embedded applications are architecture dependable applications, which has to run for the lifetime of embedded system, the offline optimization time can be considered as worth. Though compilers have employed computation and data reordering to improve locality, this still requires expert analysis due to the obscured parallelism and communication patterns in traditional 
languages such as embedded $\mathrm{C}$, embedded $\mathrm{C}++$ etc. [9, $10,14]$

Energy reduction can be achieved by exploiting the idleness of system components. Different techniques can be used to exploit the system component behavior, which is an inevitable outcome of software execution. Software controls indirectly the dynamic part of total energy consumption, which is $\mathrm{CV}^{2}$, where $\mathrm{V}$ is the operating voltage of CPU and $\mathrm{C}$ is the switching capacitance [9, 10]. At single core voltage $\mathrm{CPU}$, the compute data activity gives rise to switching capacitance; reducing this component down would decrease the energy consumption of application at its life time.

In VLIW processors, many of the components in the CPU are not completely utilized during the program execution. Primary reason for such slack is the poor architectureapplication correlation. This indicate that for an energy efficient application binary there is a need to gather more detailed profiles, containing information about system behavior on various levels (Figure 1.1). The goal of profiling is to find cause-effect relations between performance phenomena and finally generating an architecture efficient code.

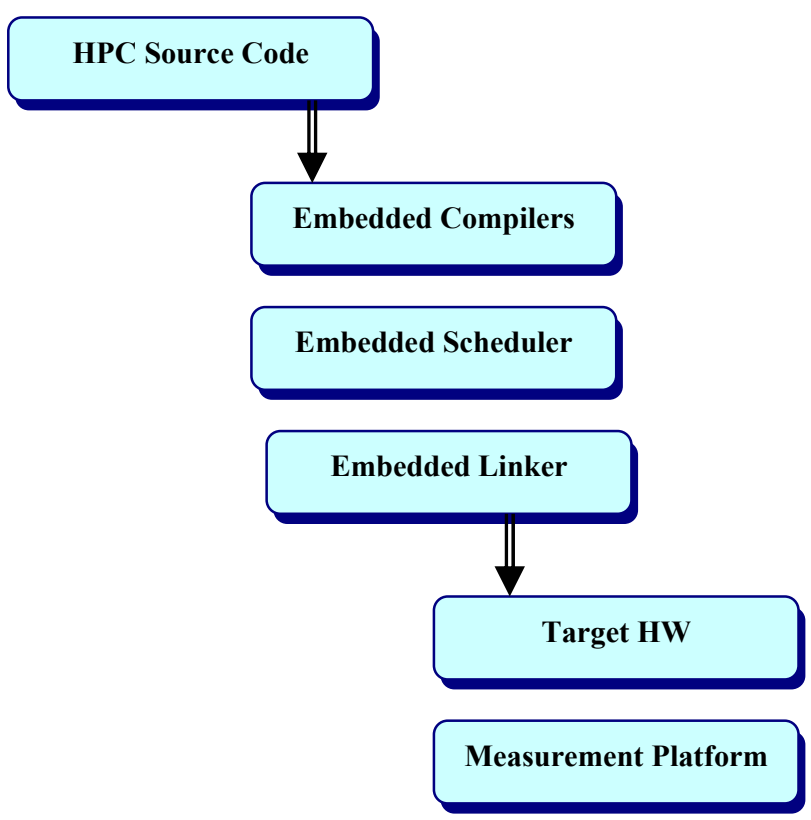

Figure 1.1. A typical HPC Application Build Flow

We add several implementations of energy efficient techniques for embedded DSP compiler to generate high performance computing applications, they are mentioned below:

- A pre-compiler based profiling, which captures application static and run time profile at all layers of application build flow. Such vertical profiling is further used to build an optimization space and finding the good one eventually.

- An iterative-compilation scheme is used further to improve the understanding of system behavior through correlation of profile information at different levels.

- A post-compiler assisted technique using a stochastic algorithm to prune huge optimization search space. It use both runtime stride and static information obtained in profiling steps.

- An architecture-based evaluation is done at real time processor and results are shown for 10 HPCs for their optimal architecture utilization.

- Results are analyzed for different architecture performance metrics such as parallelism, cache utilization, energy saving, operations per cycle. Further applications performance is evaluated for efficient architecture utilization.

The remainder of this paper is organized as follows: Target architecture and framework introduction is reviewed in Section 2. Iterative compilation and optimization strategy for our approach is presented in Section 3. The experimental setup and benchmark applications are explained in Section 4. Case studies showing the significance of our methodology are given in Section 5. Finally, conclusions are given in Section 6.

\section{Energy Aware Profiling Technique}

In this section, we will discuss how to capture the profile monitors at different layers of application build flow as shown in Figure 1. This scheme helps to obtain best performance speedup for HPC applications, but increases application size and not an appropriate choice where memory footprint is an important constraint.

\section{$2.1 \quad$ Overview}

The embedded application development life cycle starts with high level implementation of algorithm implementation as shown in Figure 1.1. Followed by is the DSP compiler for target platform. Though mostly optimization is done at the compilation level, but traditional compiler generates poor binary code, both in term of energy-cycle performance and good architectural usage. The idea in iterative compilation is to compile an application with different optimization strategies and then select the best result among these. We demonstrate that it is necessary to use an application dynamic profile at all layers to understand existing performance problems such as poor architecture usage, increased execution time, and high energy consumption. Unlike in [8], we use an entirely distinct approach to prune the optimization space. 
We consider this problem as a single task, where all desired aims have to be taken into account simultaneously. In contrast to [13], the objective function is maximized by using the genetic algorithm (GA) [11, 12]. The fitness function of a genetic algorithm represents the objective function of the underlying optimization problem and thus has an essential impact on the optimization progress of the genetic algorithm. Our energy-aware framework [6] embodies a series of profiling stages that enable the optimization process.

\subsection{StS Transformation Performance Monitors}

The accuracy of transformed code is checked against the performance of original code at target platform. The profiling stages described in Figure 1.1, detects if binary is an efficient energy application, then if needed code blocks can be restructured or transformed with transformation engine using optimal transformation scheme suggested by genetic algorithm. Followed by, basic blocks of energy-cycle critical code are located, and when necessary, converted using conventional loop optimization schemes, such as loop unrolling, loop fusion, decision tree grafting. Detail of this scheme is mentioned in [8]. Profiling is typically used to converge to optimal CPU and cache usage. In proposed framework, the impact of code transformations is fed back to transformation engine to identify performance critical bottlenecks. This mechanism requires extensive program execution analysis to get a good code. In [6], intermediate trace files are generated during the code processing flow to produce performance monitors, E.g., code size, execution time, number of cache miss, scheduling factor, and slot utilization et.. After simulation, these parameters are used to compute transformation control factors such as unrolling factor, grafting depth and blocking metrics (explained in [6]). Successively, after each cycle, each of these parameters is computed again and is compared to constraints mentioned in the user constraint file. This file contains user constraints, to be used in maximizing objective function.

\section{Compiler Assisted Stochastic Optimization (CASO)}

The overhead of finding optimal transformation space for six objectives mentioned above inevitably leads to NP hard solution. We approximate the optimization problem as the multiple objective optimization of energy saving and execution cycles. The individual candidate points in transformation space are chosen with a uniform probability distribution. They are profiled later by evaluating the application profile at the target architecture. The selected individual transformations are updated based on their success, execution cycles and energy saving factor of the sequence as a whole. Transformations contributing to better performance are rewarded while those resulting in performance losses are penalized. Thus, future sample points are more likely to include previously successful transformations more frequently and search their neighborhood more intensively.

Note that we have been using the on chip as well as off chip access metrics in our analysis as the indication to the performance. We do not account for the overlapping part for the inter-process communication and this is addressed in our next part of work, not discussed here. Our algorithm currently considers of restructuring legacy code only one source to another source at a time.

\section{Experimental Assumptions}

\subsection{Baseline Setup and Benchmarks}

We performed the experiments using the VLIW processors and its native compilation environment. The benchmark we used are 10 programs from diversified HPC domains. The important characteristics of these codes are given in $[6,8]$. Multimedia applications use DSP algorithms and streaming data schemes to compute and later to produce high throughput for real time video or audio applications. The quality of throughput depends on the application domain, e.g., bandwidth and frame rate for a typical MPEG-2 application is different at mobile device and set-top box. We chose the applications for their importance in real systems and to be representative enough to make the inferences in this study. This application set contains speech codecs (Viterbi, G-728, MP3), video codecs (MPEG-1, MPEG-2, MPEG-4), image codecs (JPEG2000) and generic DSP algorithms ( iir, fir, det, idct etc..).

\section{Results and Discussion}

The benchmarks were validated against precompiled binaries provided in the original distributions of the benchmark suites. The benchmarks were compiled at evaluation board operating at $200 \mathrm{MHz}$. The benchmark profiles were obtained using the TriMedia tmprof profiling simulator, and the performance data was collected using our framework [8].

\subsection{Sensitivity to Application-Architecture Correlation (AAC)}

Applications are the driving agent in embedded systems, and an important energy contributing factor to optimal architecture utilization. The AAC in our result is obtained by the ratio of execution of code at an infinite resource machine to the finite resource machine. For our target platform we compared it with 7- issue slot machine 
with instruction level parallel operation constraints as mentioned in [7]. Figure 5.1- Figure 5.10 shows the percentage improvement in each benchmark application to the base line code. Inherently due to highly branch oriented coding Viterbi decoder and MP3 has higher scheduling factor i.e. $17 \%$ and $20 \%$ respectively. Image compression codec JPEG2000 is dominated with deep nesting, an implicit feature of its wavelet algorithms, those results into low scheduling factor $(2 \%)$. Whereas video transcodecs reflects moderately for the scheduling factor, e.g., MPEG2 (11\%), H263 (11\%).

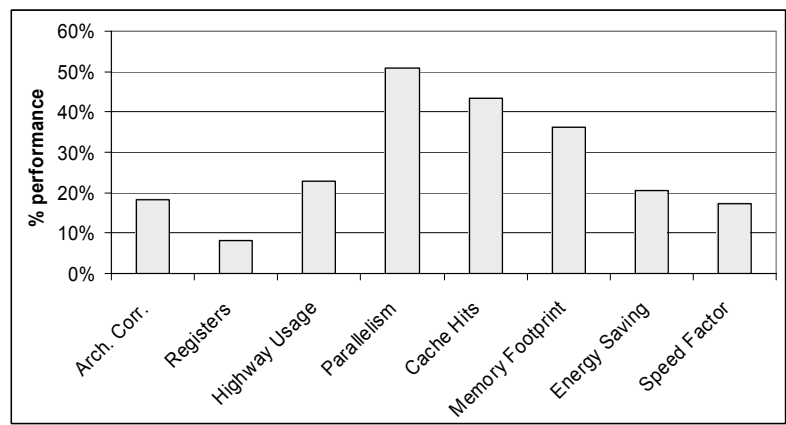

Figure 5.1. Percentage performance sensitivity in Viterbi Decoder for architecture utilization and different performance metrics as compared to their baseline version.

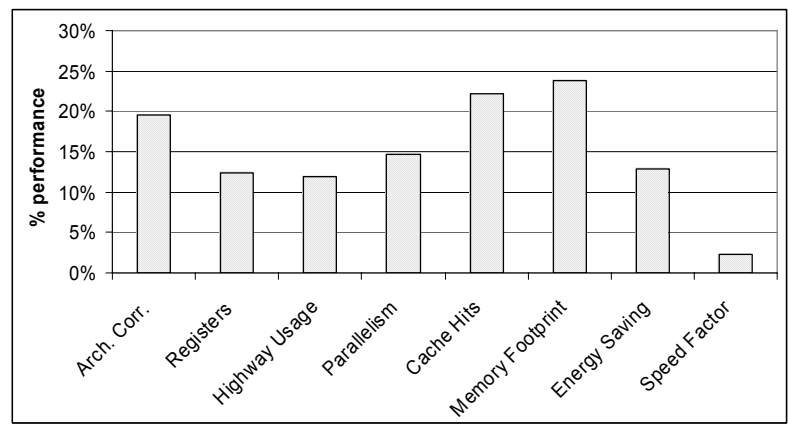

Figure 5.2. Percentage performance sensitivity in MP3 algorithm for architecture utilization and different performance metrics as compared to their baseline version.

\subsection{Parallelism Analysis}

Despite being highly localized, native compiler is inefficient to utilize on-chip register to reduce down the off-chip traffic and give rise to energy consumption as well as cycle count. E.g., for generic DSP algorithm idct (inverse direction cosine transform), the low cache hit $(12 \%)$, causes low bus activity (1\%), entailed by low anticipated scheduling factor (1\%), eventually leads to small improvement in energy saving (12\%) and reduction in execution cycles (24\%). Our hardware architecture offers high degree of parallelism, a favorite choice for applications pertaining higher temporal and spatial data independence. MPEG4, MPEG2 and JPEG2000 reflect such behavior in Figure 5.1- Figure 5.10. A careful consideration to Figure 5.1- Figure 5.10 reveals the fact that VLIW architecture is well suited for video transcodecs e.g., MPEG2. The implicit spatial and temporal parallelism in MPEG2 algorithm let it to exploit CPU 5-issue slots upto $79 \%$, raising bus activity to $22 \%$, leading to an energy as well as speed efficient application.

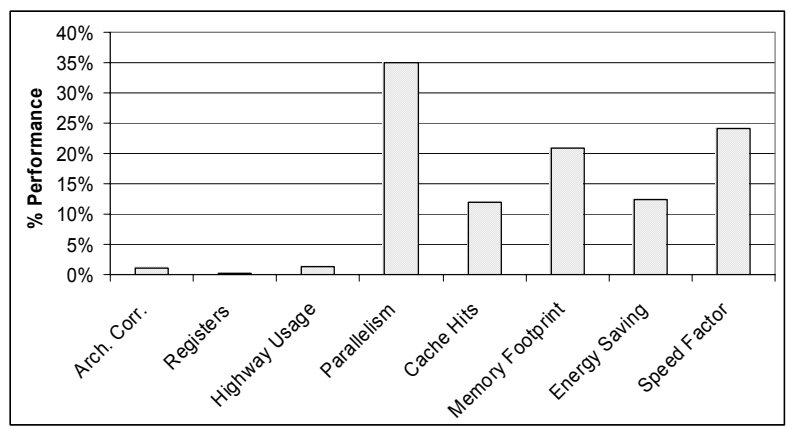

Figure 5.3. Percentage performance sensitivity in IDCT DSP algorithm for architecture utilization and different performance metrics as compared to their baseline version.

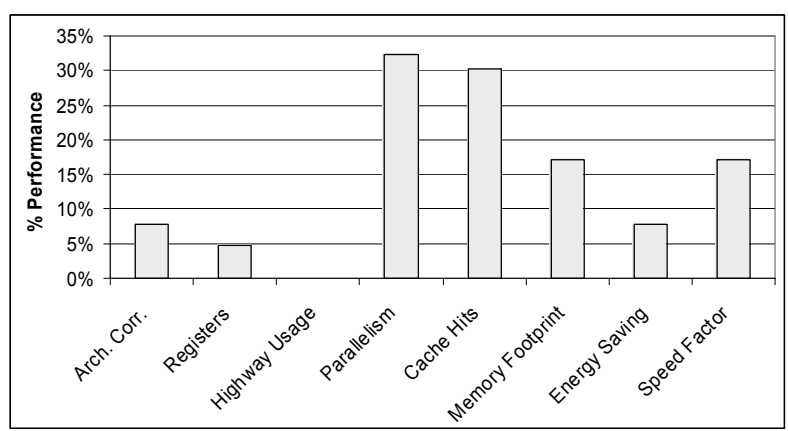

Figure 5.4. Percentage performance sensitivity in $\mathrm{AC} 3$ audio codec for architecture utilization and different performance metrics as compared to their baseline version.

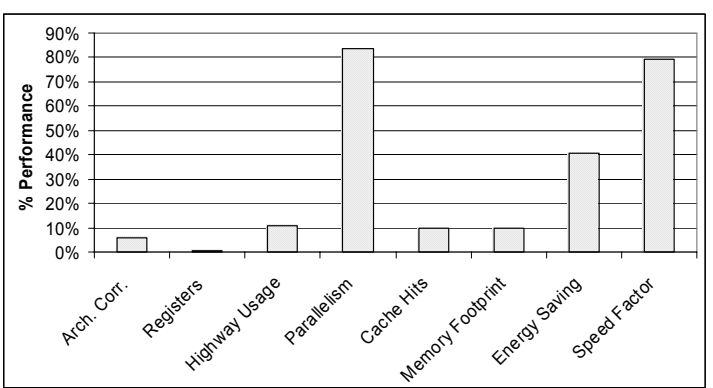

Figure 5.5. Percentage performance sensitivity in MPEG4 video codec for architecture utilization and different performance metrics as compared to their baseline version. 


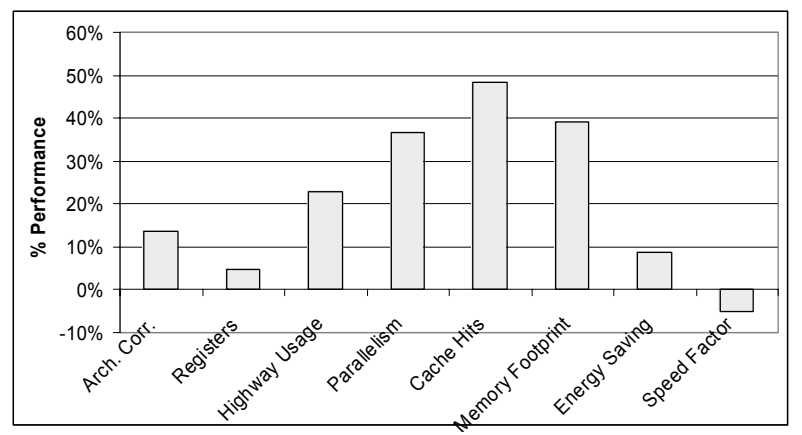

Figure 5.6. Percentage performance sensitivity in G728 Audio Codec for architecture utilization and different performance metrics as compared to their baseline version.

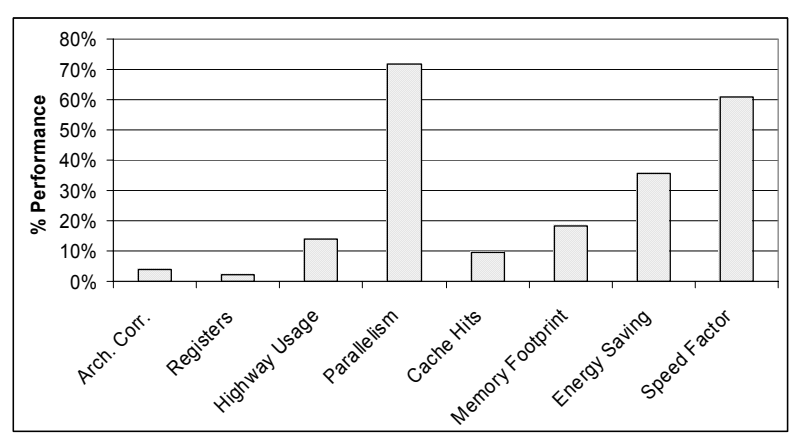

Figure 5.7. Percentage performance sensitivity in MPEG1 Video codec for architecture utilization and different performance metrics as compared to their baseline version.

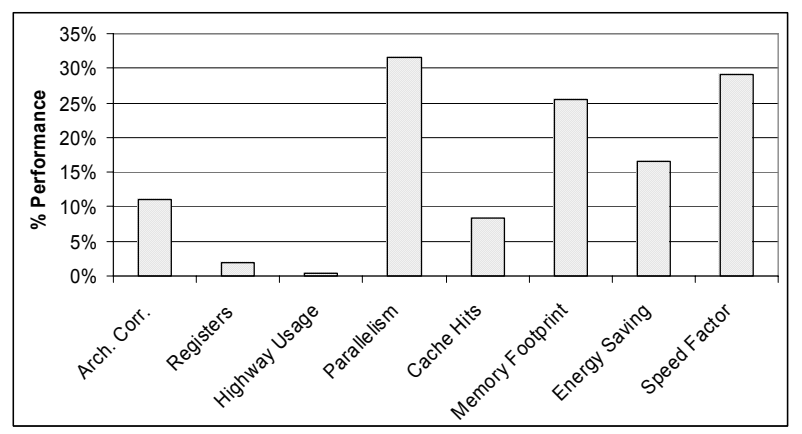

Figure 5.8. Percentage performance sensitivity in $\mathrm{H} 263$ video codec for architecture utilization and different performance metrics as compared to their baseline version.

\subsection{Energy Timing Analysis}

Primary objective of this work was two fold. First to find architecture pro applications both in term of optimization and algorithmic implementation. Second the degree of optimality that architecture provides to a candidate application for energy and cycle efficiency. Applications runtime profile in Figure 5.1- Figure 5.10, clearly conclude to the fact that there is strong correlation between the scheduling factor, cache miss, processing unit usage and speedup factor. The average cycle efficiency of video transcodecs MPEG2, MPEG1 and MPEG4 is higher than the other algorithms due to higher spatial and temporal code execution locality. While Viterbi decoder and speech codec e.g., G-728 are not suitable applications for our hardware. Second last bar graph in Figure 5.1- Figure 5.10 gives the energy saving over the baseline code for all benchmark applications. Note that MPEG1 (36\%), MPEG2 (45\%), MPEG4 (40\%), JPEG2000 (23\%) appeared as highly energy efficient application as compared to other applications in benchmark. This improvement is result of architectural utilization and we have already discussed them above.

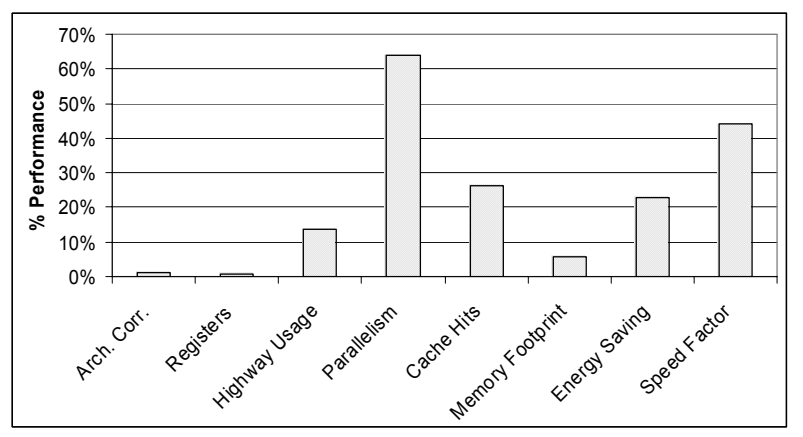

Figure 5.9. Percentage performance sensitivity in JPEG2000 image codec for architecture utilization and different performance metrics as compared to their baseline version.

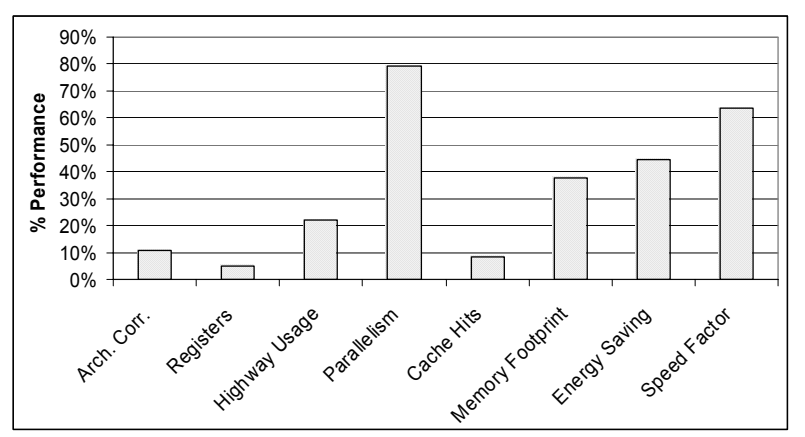

Figure 5.10. Percentage performance sensitivity in MPEG2 video codec for architecture utilization and different performance metrics as compared to their baseline version.

\section{Conclusions}

In this paper, we proposed a novel energy reduction approach for HPC execution on parallel machines. Results are compared with naïve scheme embedded in native compiler environment and optimal scheme 
integrated in native compiler. The n-iteration schemes take advantage of slacks present in conventional application build flow. The proposed scheme is applied on compute data intensive applications from different domain such as video, audio and image codecs.

The simulation results also show that a comprehensive application expression profile at all layers of application build flow can lead to better application-architecture correlation. We are investigating the proposed methodology for diversified frame based applications. In addition, we are integrating the scheme in popular DSP compilation environments. A transparent integration is the desired goal.

\section{References}

[1] Chandrakasan A. And Brodersen R. " Low Power Digital CMOS Design,” Kluwer, 1995.

[2] C. Gebotys, R. Gebotys, S. Wiratunga, "Power minimization derived from architectural-usage of VLIW processors," Proc. of the Annual ACM IEEE Design Automation Conference, pp. 308-311, June 2000.

[3] C. Gebotys, R. Gebotys, "Statistically based prediction of power dissipation for complex embedded DSP processors," Micro-processors and Microsystems Journal, vol. 23, pp. 135-144, 1999.

[4] G. Fursin, M. O’Boyle, P. Knijnenburg, "Evaluating iterative compilation," Proc. of Languages and Compilers for Parallel Computers (LCPC'02), College Park, MD, USA, 2002.

[5] V. Tiwari, S. Malik, A. Wolfe, "Compilation techniques for low energy," Proc. of the ISLPED, Oct 1994.

[6] N. Z. Azeemi, M. Rupp, "Muticriteria Low Energy Source Level Optimization of Embedded Programs," Proc. of the IEEE Informationstagung Mikroelektronik 2006, pp. 150-158, Oct. 2006.

[7] TM1300 Data Book, Philips Electronic, North America Corporation, pp. 3.1-3.16, Oct 1999.

[8] N. Zafar, M. Rupp, "Energy-aware source-to-source transformations for a VLIW DSP processor," Proc. of the IEEE $17^{\text {th }}$ ICM 2005, pp. 133-138, Dec. 2005.

[9] N. Zafar Azeemi, "Power Aware Framework for Dense Matrix Operations in Multimedia Processors," Proc. of the IEEE 9th International Multi-topic Conference, Dec. 2005.

[10] Parameswaran, S. "Code placement in hardware/software co-synthesis to improve performance and reduce cost," Proc. of the Conference on Design, Automation and Test., pp 626632, 2001.
[11] S. Bashford and R. Leupers, "Constraint driven Code Selection for Fixed-Point DSPs," Proc. of the 36th Design Automation Conference (DAC), Nov. 1999.

[12] T. Baeck. Evolutionary Algorithms in Theory and Practice. Oxford University Press, 1996

[13]N. Zafar Azeemi, "A Framework for Architecture Based Energy-Aware Code Transformations in VLIW Processors," Proc. of the IEEE International Symposium on Telecommunications (IST 2005) pp.393-398. Sep. 2005.

[14]M. Lee, V. Tiwari, S. Malik, M. Fujita, '’Power Analysis and Minimization Techniques for Embedded DSP Software,' Proc. of the IEEE Trans on VLSI Design, pp.123-135, March 1997. 